\title{
Atuação dos Centros de Atenção Psicossocial em quatro centros urbanos no Brasil
}

\author{
Rosana Teresa Onocko-Campos, ${ }^{1}$ Carlos Eduardo Menezes Amaral, ${ }^{1}$ Benedetto \\ Saraceno, ${ }^{2}$ Bruno Diniz Castro de Oliveira, ${ }^{3}$ Carlos Alberto dos Santos Treiche/ ${ }^{1}$ \\ e Pedro Gabriel Godinho Delgado 3
}

Como citar Onocko-Campos RT, Amaral CEM, Saraceno B, Oliveira BDC, Treichel CAS, Delgado PGG. Atuação dos Centros de Atenção Psicossocial em quatro centros urbanos no Brasil. Rev Panam Salud Publica. 2018;42:e113. https://doi.org/10.26633/RPSP.2018.113

RESUMO Objetivo. Descrever a atuação dos Centros de Atenção Psicossocial (CAPS) em quatro centros urbanos no Brasil.

Métodos. Realizou-se um estudo transversal (AcesSUS) com entrevista a 917 usuários acompanhados nos CAPS de Campinas, São Paulo, Porto Alegre e Fortaleza. Foram incluídas apenas as modalidades de CAPS destinadas a transtorno mental grave em adultos, sendo excluídos os CAPS voltados para uso de substâncias e para crianças e adolescentes. Os usuários responderam a questionário de múltipla escolha sobre seu percurso entre os serviços de atenção primária, CAPS e hospitais em relação aos problemas de saúde mental.

Resultados. Atendimento psiquiátrico e uso de medicação foram relatados em $90 \%$ a $100 \%$ dos usuários acompanhados nos CAPS, combinado com atendimento multiprofissional e grupos terapêuticos. O acesso à medicação foi interrompido nos 6 meses que antecederam a entrevista, especialmente em Fortaleza (64\%) e Porto Alegre (51\%). Em todos os municípios, menos de $10 \%$ dos usuários tiveram internação psiquiátrica após início do tratamento no CAPS.

Conclusões. A transição do modelo hospitalar para o comunitário continua em processo de efetivação no Brasil, com avanços comprovados. A situação, porém, é diversa nas diferentes redes de atenção, requerendo análise contextualizada de cada localidade, com identificação de dificuldades que impedem o adequado funcionamento dessas redes no paradigma psicossocial.

Palavras-chave Saúde mental; desinstitucionalização; política de saúde; inquéritos e questionários; Brasil.

No Brasil, a criação de um sistema de saúde público e universal, o Sistema Único de Saúde (SUS), a partir da

\footnotetext{
Universidade Estadual de Campinas, Departamento de Saúde Coletiva, Campinas (SP), Brasil.

2 Universidade de Genebra, Genebra, Suíça.

3 Universidade Federal do Rio de Janeiro, Instituto de Psiquiatria, Núcleo de Pesquisa em Políticas Públicas de Saúde Mental, Rio de Janeiro (RJ), Brasil. Correspondência: Bruno Diniz Castro de Oliveira, brunodinizcastro@hotmail.com
}

Constituição de 1988, contribuiu para a expansão do acesso à saúde e para a melhoria da assistência à população, com impactos positivos na expectativa de vida, mortalidade infantil, doenças infectocontagiosas e outros indicadores de saúde (1). Trata-se de um dos poucos países de grandes dimensões e renda média a ter constituído um sistema de saúde público e universal. Historicamente, decidiu-se pela constituição de um sistema de base territorial, com adscrição de população pela sua área de residência e com base na atenção primária $(\mathrm{AP})$, sendo esta a porta de entrada do sistema, organizada a partir do modelo de saúde da família.

No campo da saúde mental, desde a década de 1980, um amplo movimento social composto por profissionais de saúde, usuários dos serviços e seus familiares 
e pesquisadores tem alcançado avanços na constituição de um modelo de atenção psicossocial e comunitário, alternativo às instituições manicomiais predominantes até então. Contribuiu de forma decisiva para esses avanços a lei nacional da reforma psiquiátrica no Brasil (lei 10 216/2001) (2). Atualmente, a atenção à saúde mental consiste em uma rede de serviços que articula serviços de AP, serviços especializados de base comunitária (Centros de Atenção Psicossocial, CAPS), serviços hospitalares (com número crescente de leitos psiquiátricos em hospitais gerais e diminuição sistemática de leitos em hospitais psiquiátricos) e estratégias de desinstitucionalização, apoio social, trabalho e renda. Existem cinco modalidades de CAPS, conforme o porte (recursos humanos, dimensão física e população adscrita: CAPS I, II e III, em ordem crescente de complexidade) e o tipo de clientela atendida (adultos, crianças e adolescentes: CAPS infanto-juvenil; usuários de álcool e outras drogas: CAPS AD).

Já em 2008, publicações apontavam avanços e desafios do sistema brasileiro em relação à saúde mental. Naquela ocasião, destacavam-se como avanços a expansão de serviços comunitários e a instituição de programas de desinstitucionalização para pacientes de longa internação (3). Por outro lado, eram relatadas limitações importantes, como a distribuição desigual de serviços nas diversas regiões do país, a persistência de lacuna de tratamento associada ao incremento da prevalência de problemas de saúde mental, a insuficiente integração entre os serviços comunitários de saúde mental e a rede de atenção primária à saúde e a carência de boa formação de profissionais e gestores para a área, além da necessidade de investimento em pesquisas e da falta de um sistema de avaliação dos resultados dessa política.

Sete anos mais tarde, em 2015, um exaustivo relatório elaborado por equipe de pesquisadores internacionais convidados pela Fundação Calouste Gulbenkian, de Portugal, e Fundação Oswaldo Cruz (FIOCRUZ), do Brasil (4), destacava os mesmos avanços e a persistência de desafios, apontando algumas estratégias para a melhoria do sistema. Conforme indica o relatório, foi exitosa a transição de um cenário hospitalocêntrico para um modelo de equilíbrio, onde coexistem recursos para internação e uma rede de serviços comunitários. Embora não seja consensual, esta é a avaliação predominante (4).
Nesse contexto, o objetivo do presente artigo foi descrever os resultados de um inquérito sobre a atuação dos CAPS em quatro centros urbanos no Brasil no ano de 2016, ou seja, posteriormente à publicação do relatório Inovações e Desafios em Desinstitucionalização e Atenção Comunitária no Brasil (4).

\section{MATERIAIS E MÉTODOS}

Foram utilizados dados do estudo transversal desenvolvido no projeto AcesSUS, que analisou o acesso a partir da atenção básica, o funcionamento e a utilização da atenção especializada para hipertensão arterial grave, gravidez de alto risco, câncer de mama e transtorno grave de saúde mental em quatro municípios de grande porte: Fortaleza (estado do Ceará), Campinas e São Paulo (São Paulo) e Porto Alegre (Rio Grande do Sul) (5). O estudo transversal foi realizado entre março e setembro de 2016, com pacientes em acompanhamento nos serviços CAPS dos municípios estudados. Esses quatro municípios situam-se em diferentes regiões (Nordeste, Sudeste e Sul) e apresentam desenvolvimento desigual da implantação do SUS e da reforma psiquiátrica.

Foram incluídas apenas as modalidades de CAPS destinadas a transtorno mental grave em adultos, sendo excluídos os CAPS voltados para uso problemático de substâncias e para público infantil e adolescente. Em São Paulo, foram incluídos os 24 CAPS com maior volume de usuários e descartados os 10 serviços de menor volume. Nos demais municípios, todos os serviços foram incluídos: seis CAPS III em Campinas; cinco CAPS II e um CAPS III em Fortaleza; e quatro CAPS II em Porto Alegre.

Foram entrevistados 917 usuários dos serviços de saúde mental nos quatro municípios: 102 usuários de Campinas, 167 de Fortaleza, 351 e Porto Alegre e 297 de São Paulo. Em Campinas e Porto Alegre, optou-se por contatar todos os pacientes cadastrados nos últimos 3 anos, uma vez que esse número não era excessivo frente à capacidade operacional da pesquisa. Portanto, a abordagem dos pacientes nos CAPS com vistas ao preenchimento do questionário foi feita com base nessa listagem de pacientes, tentando-se incluir na amostra toda a população elegível independentemente do tempo dispendido na tarefa. Isso significou a permanência nos serviços por períodos de tempo distintos. Essa opção metodológica corresponde à realização de um censo de pacientes com as características de interesse em atendimento nos CAPS de Campinas e Porto Alegre. A orientação em relação à análise dos dados de saúde mental é considerá-los provenientes de levantamentos censitários. O cálculo de tamanho de amostra mínimo é justificado pela intenção de proporcionar aos pesquisadores a opção de utilizar procedimentos de inferência estatística, caso desejem.

Em São Paulo, considerando o número muito grande de serviços e pacientes, optou-se por incluir na amostra os pacientes com as características de interesse que comparecessem ao serviço para receber algum atendimento individual, em um período fixado de tempo, igual para todos os serviços.

Os critérios de inclusão foram: ter sido encaminhado ao CAPS pela AP; estar em acompanhamento há 3 anos ou menos; residir no município do serviço; apresentar condições cognitivas de responder ao questionário; e não se encontrar em crise, hospitalização psiquiátrica ou hospitalidade integral em CAPS no período da coleta de dados.

Os participantes responderam a um questionário desenvolvido na pesquisa AcesSUS, aplicado pela equipe da pesquisa nos CAPS, por meio de um aplicativo de tablet. O questionário visava a aferir informações sociodemográficas, diagnóstico autodeclarado e determinados eventos na trajetória dos usuários na rede de atenção psicossocial. As informações sociodemográficas utilizadas neste estudo incluem sexo, idade, cor, escolaridade e possuir convênio médico. O questionário foi desenvolvido especificamente para o estudo (https://www.fcm.unicamp.br/ acessus/metodologia/inquerito-amostragem/questionarios). O presente estudo enfocou os seguintes itens do questionário: intervenções recebidas no CAPS, intervenções de saúde mental na $\mathrm{AP}$, serviços buscados em situações de crise de saúde mental e internações em hospital psiquiátrico ocorridas após início do tratamento no CAPS.

A pesquisa foi aprovada nos quesitos éticos (parecer número 1654 215) e todos os participantes assinaram o TCLE.

\section{RESULTADOS}

No inquérito do projeto AcesSUS, foi possível identificar características da oferta de intervenções nos CAPS de quatro 
grandes municípios que asseguram a efetividade desses serviços. Identificou-se que nos CAPS de Campinas, Fortaleza, Porto Alegre e São Paulo, 95 (93\%), 154 (92\%), 348 (99\%) e 280 (94\%) usuários eram atendidos individualmente por psiquiatras, respectivamente. Já quanto a atendimentos individualizados por profissionais de outras categorias (assistente social, enfermeiro, psicólogo ou terapeuta ocupacional), identificou-se que nos CAPS de Campinas, Fortaleza, Porto Alegre e São Paulo, 102 (100\%), 75 (45\%), 256 $(73 \%)$ e $267(90 \%)$ usuários relataram ter passado por algum atendimento desse tipo.

Em todos os municípios, ampla maioria dos usuários recebeu medicação psicotrópica. Em Fortaleza e São Paulo, 152 (92\%) e 265 (91\%) usuários referiram recebimento de medicação, respectivamente. Nos municípios de Porto Alegre e Campinas, 331 (95\%) e 100 (99\%) usuários referiram recebimento de medicação, respectivamente. Apesar de os dados acerca do recebimento de medicação no CAPS sugerirem que a maior parte dos usuários estivesse fazendo uso de algum psicotrópico, boa parte relatou dificuldades de acesso à medicação. Nos 6 meses que antecederam a entrevista, houve interrupções no fornecimento, especialmente no município de Fortaleza, relatada por 105 (64\%) usuários; para 92 usuários (58\%), a interrupção ocorreu em mais de uma ocasião. Em Porto Alegre, 168 (51\%) usuários relataram interrupção do fornecimento de medicação, sendo $136(40 \%)$ relatos de interrupção em mais de uma ocasião.

Além do atendimento individual e tratamento farmacológico, a maioria dos usuários de CAPS referiu ter recebido outras formas de tratamento (tabela 1). Receberam ofertas de outras formas de tratamento em Campinas, Porto Alegre e São Paulo, respectivamente, 95 (93\%), $314(89 \%)$ e $235(80 \%)$ usuários. Houve uma menor proporção desse tipo de oferta em Fortaleza: 55\% $(\mathrm{n}=90)$. A forma mais frequente de intervenção foram os grupos realizados no próprio CAPS, destacando-se Porto Alegre (tabela 1).

A maioria dos usuários estudados recebeu atendimento pelas equipes da AP: 100 (98\%) em Campinas, 151 (90\%) em Fortaleza, 346 (99\%) em Porto Alegre e 276 (94\%) em São Paulo. Intervenções incluindo tratamento em saúde mental na atenção básica foram relatadas por
72 (71\%) usuários em Campinas, 78 (47\%) em Fortaleza, 247 (70\%) em Porto Alegre e 193 (65\%) em São Paulo.

A atenção à crise apresenta variações importantes entre os municípios pesquisados. Em Campinas, destaca-se o papel dos CAPS, com 74 (73\%) usuários reportando buscar o serviço. Em São Paulo, o CAPS também foi o serviço mais procurado, porém com percentual mais modesto: 39\% $(n=113)$. Em Fortaleza e Porto Alegre, esse serviço foi mencionado por menos de um terço dos entrevistados ( $n=33,20 \%$; e $n=92,27 \%$, respectivamente). Em Fortaleza, o serviço mais procurado foi o hospital psiquiátrico ( $n=46,28 \%$ ), ao passo que em Porto Alegre destacou-se o contato com a atenção básica ( $\mathrm{n}=110,31 \%$ ) e com os serviços de emergência geral ( $\mathrm{n}=68,19 \%$ ). O caráter substitutivo da rede CAPS de Campinas e São Paulo é destacado também pela procura infrequente por hospitais psiquiátricos - zero e $23(7,9 \%)$ usuários, respectivamente.

Em todos os municípios, menos de $10 \%$ dos usuários tiveram internação psiquiátrica após início do tratamento no CAPS (tabela 2). Menos de $1 \%$ tiveram mais de quatro internações nos quatro municípios. Cabe destacar que os usuários entrevistados se encontravam em tratamento no CAPS por um período igual ou menor que 3 anos. A tabela 2 mostra as informações quanto aos serviços procurados em situação de crise e o número de internações em hospital psiquiátrico após início do tratamento no CAPS.

\section{DISCUSSÃO}

Os presentes resultados oferecem evidência de que o Brasil conseguiu enfrentar, ainda que parcialmente, o desafio de transformar a saúde mental, propondo e implantando tecnologias novas, como

TABELA 1. Formas de tratamento oferecidas nos Centros de Atendimento Psicossocial (CAPS) de Campinas, Fortaleza, Porto Alegre e São Paulo, 2015

\begin{tabular}{|c|c|c|c|c|c|c|c|c|}
\hline \multirow[t]{2}{*}{ Forma de tratamento } & \multicolumn{2}{|c|}{$\begin{array}{l}\text { Campinas } \\
(\mathrm{n}=102)\end{array}$} & \multicolumn{2}{|c|}{$\begin{array}{l}\text { Fortaleza } \\
(n=167)\end{array}$} & \multicolumn{2}{|c|}{$\begin{array}{l}\text { Porto Alegre } \\
\qquad(\mathrm{n}=351)\end{array}$} & \multicolumn{2}{|c|}{$\begin{array}{l}\text { São Paulo } \\
(n=297)\end{array}$} \\
\hline & $\mathrm{n}$ & $\%$ & $n$ & $\%$ & $n$ & $\%$ & $\mathrm{n}$ & $\%$ \\
\hline Atendimento individual com psiquiatra & 95 & 93 & 154 & 92 & 348 & 99,2 & 280 & 94,3 \\
\hline $\begin{array}{l}\text { Atendimento individual com outro } \\
\text { profissional }^{\mathrm{a}}\end{array}$ & 102 & 100 & 75 & 44,9 & 256 & 72,9 & 267 & 89,9 \\
\hline Tratamento psicotrópico & 100 & 99 & 152 & 92 & 331 & 95 & 265 & 91 \\
\hline Passar o dia no CAPS & 29 & 28 & 0 & 0 & 96 & 27 & 82 & 28 \\
\hline Visitas domiciliares & 7 & 7 & 0 & 0 & 31 & 9 & 13 & 4 \\
\hline Grupos no CAPS & 57 & 56 & 43 & 26 & 281 & 80 & 189 & 65 \\
\hline Grupos na comunidade & 11 & 11 & 13 & 8 & 10 & 3 & 12 & 4 \\
\hline Acolhimento em leito CAPS & 16 & 16 & 0 & 0 & 1 & 0 & 1 & 0 \\
\hline
\end{tabular}

a Profissional de saúde de nível superior: assistente social, enfermeiro, psicólogo ou terapeuta ocupacional.

TABELA 2. Serviços de saúde mental buscados pelos usuários em situações de crise e internações em hospital psiquiátrico, Campinas, Fortaleza, Porto Alegre e São Paulo, 2015

\begin{tabular}{|c|c|c|c|c|c|c|c|c|}
\hline \multirow[t]{2}{*}{ Variável } & \multicolumn{2}{|c|}{$\begin{array}{l}\text { Campinas } \\
(n=102)\end{array}$} & \multicolumn{2}{|c|}{$\begin{array}{l}\text { Fortaleza } \\
(n=167)\end{array}$} & \multicolumn{2}{|c|}{$\begin{array}{l}\text { Porto Alegre } \\
(n=351)\end{array}$} & \multicolumn{2}{|c|}{$\begin{array}{l}\text { São Paulo } \\
(n=297)\end{array}$} \\
\hline & $n$ & $\%$ & $n$ & $\%$ & $\mathrm{n}$ & $\%$ & $n$ & $\%$ \\
\hline \multicolumn{9}{|l|}{ Serviço ${ }^{a}$} \\
\hline CAPS & 74 & 73 & 33 & 20 & 92 & 27 & 113 & 39 \\
\hline Atenção básica & 14 & 14 & 4 & 2 & 110 & 32 & 30 & 10 \\
\hline Pronto socorro & 16 & 16 & 11 & 7 & 68 & 20 & 50 & 17 \\
\hline Hospital geral & 10 & 10 & 11 & 7 & 30 & 9 & 45 & 16 \\
\hline Hospital psiquiátrico & 0 & 0 & 46 & 28 & 35 & 10 & 23 & 8 \\
\hline \multicolumn{9}{|l|}{ Número de internações } \\
\hline Nenhuma & 95 & 94 & 160 & 96 & 328 & 93 & 282 & 95 \\
\hline 1 a 4 internações & 6 & 6 & 7 & 4 & 22 & 6 & 12 & 4 \\
\hline 5 ou mais internações & 0 & 0 & 0 & 0 & 1 & 0 & 3 & 1 \\
\hline
\end{tabular}

a Mais de um serviço poderia ser citado. 
os CAPS. Como já apontado no relatório Inovações e Desafios em Desinstitucionalização e Atenção Comunitária no Brasil (4), permaneceu em 2015 a trajetória de expansão da rede de CAPS, que atingiu $67 \%$ da cobertura ideal, ao mesmo tempo em que houve progressiva diminuição no número de leitos psiquiátricos (figura 1). Contudo, apesar da inversão no financiamento entre 2002 e 2013, com declínio nos gastos com hospitais e crescimento contínuo dos gastos com serviços comunitários (4), o financiamento dirigido aos serviços comunitários ainda é insuficiente. O país investe cerca de $2,5 \%$ dos recursos totais da saúde em saúde mental, proporção insuficiente diante da magnitude epidemiológica e complexidade do problema. Conforme o relatório, existem precariedades importantes na estrutura dos serviços comunitários, especialmente quanto à área física, insumos e pequeno número de serviços para crianças e adolescentes (4). Deve ser ressaltado que, a partir de 2016, a política de saúde pública brasileira tem sofrido redução drástica de financiamento e retrocesso no ritmo de implantação de novos serviços, em consequência da mudança nos rumos da política nacional no nível federal e pela crise financeira que assolou estados e municípios (6).

No estudo AcesSUS, o modelo de atendimento observado indica ênfase e variedade no atendimento clínico dos

FIGURA 1. Criação de Centros de Atendimento Psicossocial (CAPS) e declínio dos leitos psiquiátricos no Brasil, 2001 a 2015a

(a)

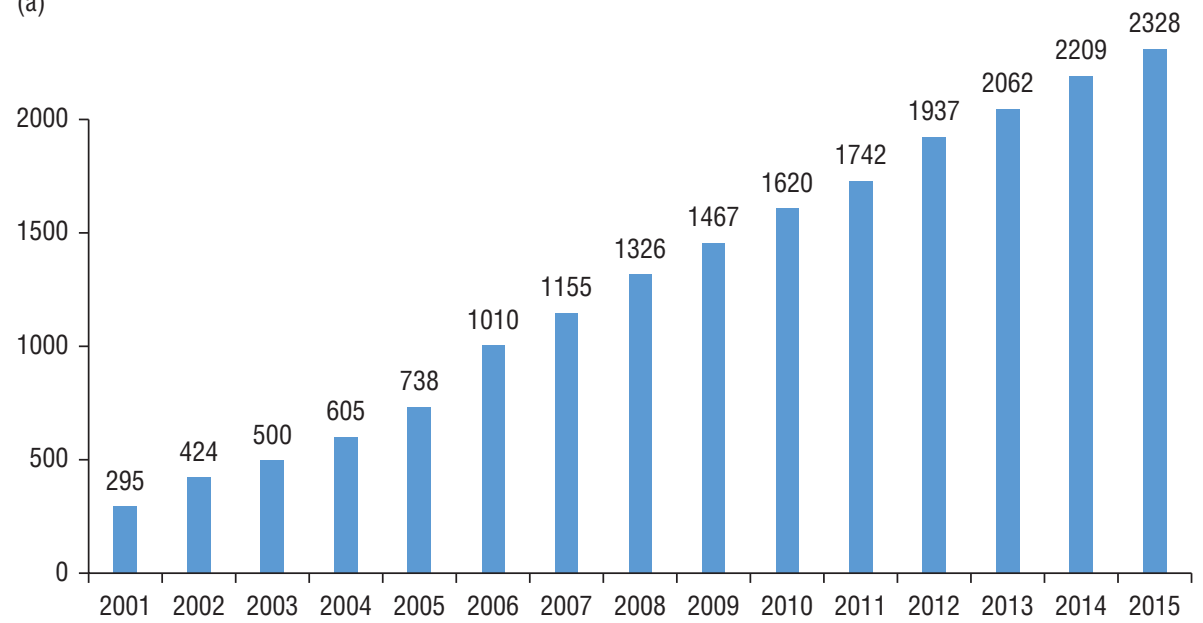

(b)

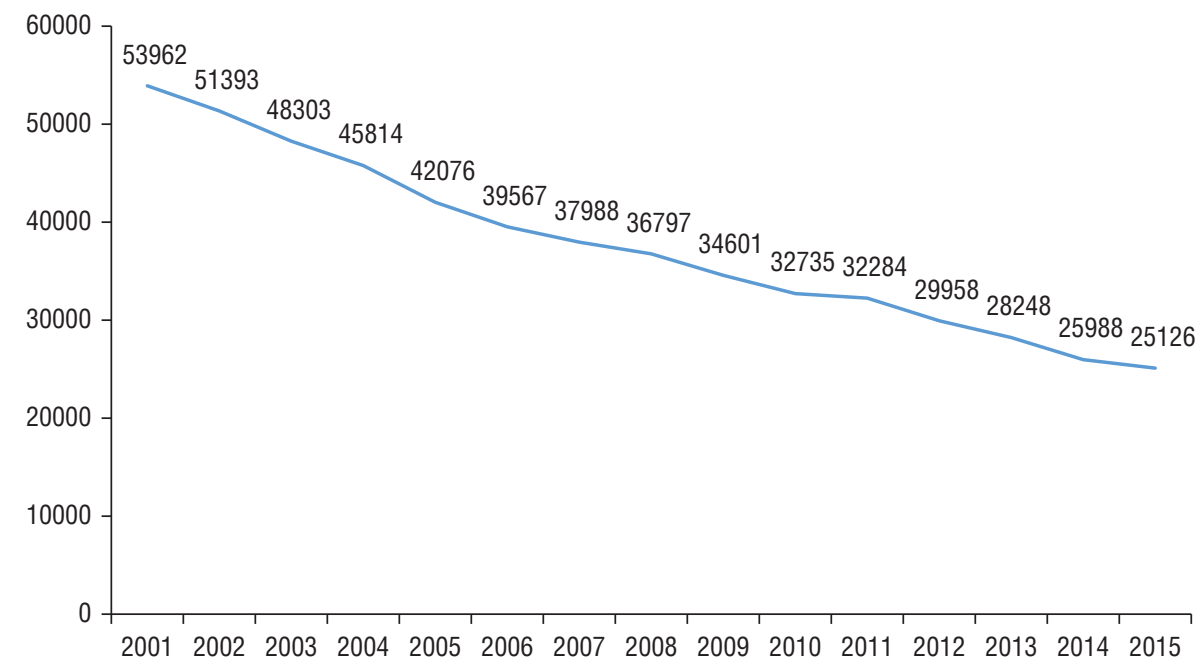

Fonte: Ministério da Saúde, 2015. Adaptado de Fundação Oswaldo Cruz, Fundação Calouste Gulbenkian (4). a) Número de CAPS; B) número de leitos psiquiátricos. usuários. A grande maioria dos usuários dos serviços utilizou consultas e medicações psiquiátricas, resultado convergente com outros estudos (7). Porém, cabe destacar que houve oferta considerável de opções terapêuticas para além da medicação, atestando o afastamento dos serviços comunitários de saúde mental do modelo ambulatorial biomédico.

Identificou-se alta proporção de usuários em cada município com consultas com outros profissionais de saúde ou participação em grupos no serviço, indicando a atuação multiprofissional. Somente o modelo observado em Fortaleza destoou dos demais municípios: houve concentração no atendimento individual de psiquiatria, semelhante aos outros municípios porém com pequena oferta de atendimento multiprofissional e baixa utilização de outros recursos como grupos, visitas domiciliares e outras atividades no CAPS. Ainda que inovações tenham ocorrido na saúde mental de Fortaleza (8), de modo geral o modelo fortalezense parece manter uma postura reducionista do cuidado, conforme identificado em estudos anteriores (9).

As diferenças entre municípios evidenciam propostas de cuidado com ênfases distintas. Em Campinas destaca-se a utilização dos leitos dos CAPS III. Entretanto, este recurso é pouquíssimo utilizado nos demais municípios, que contam com cobertura insuficiente de serviços CAPS III, sugerindo dificuldades na atenção à crise sem o recurso ao hospital. A oferta de ação conjunta entre CAPS e hospital geral ou psiquiátrico foi pouco mencionada, indicando uma provável ruptura da continuidade do cuidado e vínculo com a equipe do CAPS nas situações de internação.

Campinas e Fortaleza também se destacam pela proporção de grupos realizados fora do serviço, o que sugere que parcerias e inserção comunitária têm sido melhor desenvolvidas nessas localidades. A atuação comunitária é um importante aspecto no desenvolvimento da dimensão sociocultural da Reforma Psiquiátrica (10), promovendo a circulação social dos usuários para além da rede formal de serviços.

Deficiência importante e recorrente refere-se à falta de medicação. Em todos os municípios houve proporção significativa de pessoas que sofreram interrupção da oferta da medicação prescrita em algum momento dos últimos 6 meses, evidenciando financiamento insuficiente 
e dificuldades logísticas na dispensação farmacêutica.

A maior parte dos usuários do CAPS oriundos da AP recebeu intervenções de saúde mental com uso de medicação, o que aponta para os psicotrópicos como um recurso central das ações na AP. Estudos anteriores sobre o cuidado em saúde mental na atenção básica no Brasil (11) apontavam para ações das equipes generalistas baseadas em aconselhamento, senso comum ou encaminhamento instantâneo de quaisquer demandas $(12,13)$, ao passo que estudos mais recentes destacam a qualificação da prescrição medicamentosa a partir do apoio matricial (14). Contudo, a centralidade da medicação é relativizada, segundo estudos que relatam o fortalecimento do uso de outros dispositivos pelas equipes de atenção básica, tais como grupos, atuação dos agentes comunitários de saúde, uso de recursos comunitários, articulações intersetoriais e promoção de autocuidado (14, 15-17). Uma hipótese para a aparente centralidade da medicação identificada em nosso estudo seria a dificuldade de os usuários identificarem tais recursos alternativos como "ações de saúde mental", de modo a não os incluir no relato de "tratamento" recebido na atenção básica.

Campinas apresentou a maior proporção de usuários que receberam intervenções de saúde mental na AP sem uso de medicação. Ainda que as equipes desse município possuam maior presença de psiquiatras na AP do que os demais municípios, a composição dessas equipes, que contam com psicólogo e terapeuta ocupacional, parece promover uma oferta de cuidado menos medicalizante.

Os serviços CAPS apresentaram boa aceitação na atenção à crise. Fortaleza destoou dos demais municípios pela manutenção da referência principal no hospital psiquiátrico, ainda que a procura pelo CAPS seja semelhante à de Porto Alegre. Porto Alegre também destoou das demais localidades por alta procura pela AP na crise, o que parece determinado pela política municipal que estabelece a regulação do fluxo pela AP.

A resposta à crise demanda uma intencionalidade por parte dos serviços territoriais (18), assim como uma consideração dos diversos significados atribuídos pelos atores: profissionais, usuários, familiares e comunidade (19). Nesse contexto complexo de resposta às crises, a capilaridade dos CAPS na rede social e técnica de cada localidade é crucial. A pequena disponibilidade de CAPS III nos municípios diminui a efetividade da rede de atenção, com risco de retorno às práticas segregadoras que ocorriam em instituições psiquiátricas.

Os usuários de todos os municípios apresentaram baixa frequência de internações psiquiátricas. Todavia, destaca-se que Campinas, apesar da baixa procura pelo hospital psiquiátrico nas crises, apresentou uma proporção de usuários com histórico de internações semelhante aos demais municípios. Fortaleza, apesar da procura preferencial dos usuários pelo hospital psiquiátrico nas situações de crise, apresentou uma proporção semelhante aos demais municípios de usuários já internados. Em Campinas, a baixa procura pelo hospital foi convergente com o histórico investimento do município na Reforma psiquiátrica.

A literatura mostra que as regiões Sul e Sudeste contam com maior capacidade econômica e melhor acesso aos serviços de saúde, ao passo que as regiões Norte e Nordeste possuem piores indicadores de morbimortalidade e socioeconômicos (20). Da mesma forma, a implantação das redes assistenciais ainda está em desenvolvimento, apresentando estágios de consolidação e inovação diversificados nos diferentes estados e municípios brasileiros (21). Contudo, não existem avaliações sistemáticas sobre o funcionamento dessas redes. Na saúde mental, as avaliações e pesquisas ainda se concentram na análise de serviços isolados $(22,23)$ e dos CAPS, assim como na investigação da articulação desses serviços com a $\operatorname{AP}(14,15,24)$. Sendo assim, são recomendáveis novos estudos que avaliem a interlocução com outros serviços, assim como as dimensões avaliativas envolvidas nas redes $(21,25)$.

São limitações do presente estudo a inclusão de apenas quatro centro urbanos, que não contemplam todas as regiões brasileiras, e as clássicas limitações dos estudos transversais. Contudo, dada a escassez de estudos sobre o tema, ele possui como ponto forte se constituir em uma primeira aproximação à produção dos CAPS nos grandes centros urbanos brasileiros.

\section{CONCLUSÕES}

A transição do modelo hospitalar para o comunitário continua em processo de efetivação no Brasil, com avanços comprovados. A situação, porém, é diversa nas diferentes redes de atenção, requerendo análise contextualizada de cada localidade, identificando os pontos de dificuldades que impedem o adequado funcionamento destas no paradigma psicossocial.

O financiamento torna-se um desafio crucial, especialmente por conta das decisões do novo governo brasileiro, congelando por 20 anos os gastos com saúde e outras políticas sociais (26), e das recentes normativas do Ministério da Saúde que redirecionam os recursos financeiros para os hospitais e comunidades terapêuticas (27). No período estudado, a mudança dos recursos financeiros, do componente hospitalar para as ações comunitárias, foi uma característica estrutural marcante e decisiva do processo brasileiro de reforma psiquiátrica (28).

Assim, estamos diante de uma ameaça concreta de retrocesso na política de saúde mental comunitária. Os avanços conquistados em relação à AP devem-se fundamentalmente ao apoio dos profissionais de saúde mental, realizadas no âmbito da Estratégia Saúde da Família. A nova Política de Atenção Básica (29), também aprovada pelo atual governo federal, fragiliza o modelo ao flexibilizar formas de contratação sem a constituição adequada das equipes multiprofissionais, apresentando outra ameaça ao modelo de atenção à saúde mental.

A rede de serviços comunitários implantada está presente em todas as regiões do país, e significou uma real ampliação do acesso ao tratamento. A sustentabilidade e fortalecimento dessa imensa rede de serviços é o grande desafio da política de saúde mental no Brasil hoje, assim como o combate às iniquidades regionais que ainda persistem.

Agradecimentos. O trabalho foi financiado pelo Conselho Nacional de Desenvolvimento Científico e Tecnológico (CNPq), processo $n^{\circ} 405044 / 2013-5$, no âmbito da Chamada MCTI/CNPq/CT Saúde/MS/SCTIE/Decit N 41/2013 Rede Nacional de Pesquisas sobre Política de Saúde: Conhecimento para Efetivação do Direito Universal à Saúde.

Conflitos de interesse. Nada declarado pelos autores.

Declaração. As opiniões expressas no manuscrito são de responsabilidade exclusiva dos autores e não refletem necessariamente a opinião ou política da RPSP/PAJPH ou da Organização Pan-Americana da Saúde (OPAS). 


\section{REFERÊNCIAS}

1. Victora CG, Barreto ML, do Carmo Leal M, Monteiro CA, Schmidt MI, Paim J, et al. Health conditions and health-policy innovations in Brazil: the way forward. Lancet. 2011; 4;377(9782):2042-53.

2. Delgado PGG. Saúde mental e direitos humanos: 10 anos da Lei 10.216/2001. Arq Bras Psicol. 2011;63(2):114-21.

3. Mateus MD, Mari JJ, Delgado PG, AlmeidaFilho N, Barrett T, Gerolin J, et al. The mental health system in Brazil: Policies and future challenges. Int J Ment Health Syst. 2008;2(1):12.

4. Fundação Oswaldo Cruz, Fundação Calouste Gulbenkian. Inovações e Desafios em Desinstitucionalização e Atenção Comunitária no Brasil. Seminário Internacional de Saúde Mental: Documento Técnico Final. Fiocruz, Fundação Calouste Gulbenkian, Organização Mundial de Saúde, Ministério da Saúde; 2015. Disponível em: http:/ / www.gulbenkianmhplatform.com/conteudos/00/61/00/04/ Desinstitucionaliza\%C3\%A7\%C3\%A3o\&-aten $\%$ C $3 \%$ A $7 \%$ C $3 \%$ A3o-comunit $\%$ C $3 \%$ A 1ria.Fiocruz-Gulbenkian.Documento---t\%C3\%A9cnico.Mar\%C3\% A7o2015_4416.pdf Acessado em junho de 2018.

5. AcesSUS. Disponível em: https://www. fcm.unicamp.br/acessus/ Acessado em junho de 2018.

6. Vieira FS, Benevides RPS. Os impactos do novo regime fiscal para o financiamento do Sistema Único de Saúde e para a efetivação do direito à saúde no Brasil. (Nota técnica $\mathrm{n}^{\circ}$ 28). Instituto de Pesquisa Econômica Aplicada (IPEA). Brasília: IPEA; 2016. Disponível em: http://www.ipea.gov.br/ portal / index.php?option $=$ com content\&id=28589 Acessado em junho de 2018.

7. Kantorski LP, Jardim VM da R, Porto AR, Schek G, Cortes JM, Oliveira MM de. Descrição de oferta e consumo dos psicofármacos em Centros de Atenção Psicossocial na Região Sul brasileira. Rev Esc Enferm USP. 2011;45(6):1481-7.

8. Bosi ML, Carvalho LB, Ximenes VM, Melo AK da S, Godoy MGC. Inovação em saúde mental sob a ótica de usuários de um movimento comunitário no nordeste do Brasil Innovation in mental health from the perspective of users: a community movement in the Northeast of Brazil. Cienc Saude Colet. 2012;17(3):643-51.

9. Quinderé PHD, Sales F, Albuquerque R, Jorge MSB. A convivência entre o modelo psicossocial e asilar em fortaleza. Saede Debate. 2010;34(8):137-47.

10. Amarante P. Reforma psiquiátrica e epistemologia. Cad Bras Saude Ment. 2009;1(1).

11. Campos GW de S. Equipes de referência e apoio especializado matricial : um ensaio sobre a reorganização do trabalho em saúde. Cienc Saude Colet. 1999;4(2):393-403.

12. Arce VAR, Sousa MF de, Lima M da G. A práxis da Saúde Mental no âmbito da Estratégia Saúde da Família: contribuições para a construção de um cuidado integrado. Physis. 2011;21(2):541-60.

13. Lucchese R, Oliveira AGB de, Conciani ME, Marcon SR. Saúde mental no Programa Saúde da Família: caminhos e impasses de uma trajetória necessária. Cad Saude Publica. 2009;25(9):2033-42.

14. Quinderé PHD, Jorge MSB, Nogueira MSL, Costa LFA da, Vasconcelos MGF. Acessibilidade e resolubilidade da assistência em saúde mental: a experiência do apoio matricial. Cienc Saude Coletiva. 2013;18(7):2157-66.

15. Figueiredo MD, Onocko-Campos R. Saúde Mental na atenção básica à saúde de Campinas, SP: uma rede ou um emaranhado? Cienc Saude Coletiva. 2009;14(1): 129-38.

16. Onocko-Campos R, Gama CA, Ferrer AL, Santos DVD dos, Stefanello S, Trapé TL, et al. Saúde mental na atenção primária à saúde: estudo avaliativo em uma grande cidade brasileira. Cienc Saude Coletiva. 2011;16(12):4643-52.

17. Amaral CEM, Moreira CP, Nunes $\mathrm{M}$ de O, Torrenté $\mathrm{M}$ de. Apoio matricial em saúde mental na atenção básica: efeitos na compreensão e manejo por parte de Agentes Comunitários de Saúde. Interface. 2018; 22(67).

18. Dell'Acqua G, Mezzina R. Resposta à crise: estratégia e intencionalidade da intervenção no serviço psiquiátrico territorial. Em: Amarante P, editor. Arquivos de Saúde Mental e Atenção Psicossocial 2. Rio de Janeiro: Nau; 2005. Pp. 161-94.

19. Lima M, Jucá VJ dos S, Nunes M de O, Ottoni V. Signos, significados e práticas de manejo da crise. Interface. 2012;16(41): 423-34.

20. Monteiro Neto A. Desigualdades regionais no Brasil: características e tendências recentes. Boletim Regional Urbano Ambiental. 2014;9:97-81.

21. Hartz Z, Contandriopoulos A. Integralidade da atenção e integração de serviços de saúde: desafios para avaliar a implantação de um "sistema sem muros." Cad Saude Publica. 2004;20(2):331-6.

22. Dantas CR, Oda AMGR. Mapping of inquiries in mental health care in Brazil (2004-2013). Physis. 2014;24(4):1127-79.

23. Costa PHA da, Colugnati FAB, Ronzani TM. Avaliação de serviços em saúde mental no Brasil: revisão sistemática da literatura. Cienc Saude Coletiva. 2015;20(10): 3243-53.

24. Sousa FSP de, Jorge MSB, Vasconcelos MGF, Barros MMM de, Quinderé PHD Gondim LGF. Tecendo a rede assistencial em saúde mental com a ferramenta matricial. Physis. 2011;21(4):1579-99.

25. Amaral CEM, Bosi MLM. Network as transconcept: elements for a conceptual demarcation in the field of public health. Rev Saude Publica. 2016;22;50:51.

26. Brasil. Emenda Constitucional 95/2016. Disponível em: http:/ /legis.senado.leg.br/ legislacao $/$ DetalhaSigen .action :id $=540698$ Acessado em junho de 2018.

27. Ministério da Saúde, Comissão Intergestores Tripartite. Resolução 32/2017. Disponível em: http://portalarquivos2.saude.gov.br/ images/pdf/2018/janeiro/05/Resolu-----oCIT-n---32.pdf Acessado em junho de 2018.

28. Gonçalves RW, Vieira FS, Delgado PGG. Política de Saúde Mental no Brasil: evolução do gasto federal entre 2001 e 2009. Rev Saude Publica. 2012;46(1):51-8.

29. Ministério da Saúde. Portaria 2 436/2017. Disponível em: http://bvsms.saude.gov. br / bvs / saudelegis / g m / 2017 / MatrizesConsolidacao/comum/250584. html Acessado em junho de 2018.

Manuscrito recebido em 13 de março de 2018. Aceito em versão revisada em 22 de maio de 2018. 
ABSTRACT

\section{Functioning of Psychosocial Care centers in four cities in Brazil}

Objective. To describe the functioning of Psychosocial Care centers (CAPS) in four Brazilian cities.

Methods. In this cross-sectional study (AcesSUS), 917 CAPS users were interviewed in the cities of Campinas, São Paulo, Porto Alegre, and Fortaleza. Only CAPS focused on severe mental disorders in adults were included, that is, CAPS focusing on substance abuse and on children and adolescents were excluded. Users answered a multiple choice questionnaire about their path from primary health care services until reaching the CAPS and hospital care in relation to mental health problems.

Results. Care by a psychiatrist and use of medications were reported by $90.7 \%$ to $99.7 \%$ of CAPS users in association with multiprofessinal care and therapeutic groups. Access to medication was interrupted in the 6 months prior to the interview, especially in Fortaleza (70\%) and Porto Alegre (50\%). In all cities, less than $10 \%$ of users had a psychiatric hospital admission after treatment at the CAPS was started.

Conclusions. The transition from a hospital-centered model to a community-based model is well under way in Brazil, with concrete achievements. However, there is heterogeneity among different care networks, which entails the need for contextualized analysis of each individual location, with identification of difficulties that prevent the adequate functioning of these care networks in the psychosocial paradigm.

Keywords Mental health; deinstitutionalization; health policy; surveys and questionnaires; Brazil.

RESUMEN Objetivo. Describir la actuación de los Centros de Atención Psicosocial (CAPS) en cuatro centros urbanos en Brasil.

Métodos. Se realizó un estudio transversal (AcesSUS) por medio de una entrevista a 917 usuarios en seguimiento en los CAPS de Campinas, São Paulo, Porto Alegre y

Actuación de los Centros de Atención Psicosocial en cuatro centros urbanos en Brasil graves en adultos, con exclusión de los centrados en el uso de sustancias y servicios para niños y adolescentes. Los usuarios respondieron a un cuestionario de opción múltiple sobre su recorrido entre los servicios de atención primaria, los CAPS y los hospitales en relación con los trastornos de salud mental.

Resultados. Se notificaron servicios de atención psiquiátrica y uso de medicamentos, junto con atención multiprofesional y participación en grupos terapéuticos, en una proporción de $90,7 \%$ a $99,7 \%$ de los usuarios en seguimiento en los CAPS. El acceso a medicamentos se interrumpió en los 6 meses anteriores a la entrevista, particularmente en Fortaleza (70\%) y Porto Alegre (50\%). En todos los municipios, menos de $10 \%$ de los usuarios estuvieron internados en instituciones psiquiátricas después de iniciar el tratamiento en los CAPS.

Conclusiones. La transición del modelo hospitalario al comunitario sigue en curso de implementación en Brasil, con adelantos comprobados. Sin embargo, la situación es diversa en las diferentes redes de atención, lo cual exige un análisis contextualizado de cada localidad y la determinación de las dificultades que impiden el funcionamiento adecuado de esas redes en el paradigma psicosocial. 